\title{
Post an die Kasse nach EBM abrechnen, Patientenbrief höchstens nach GOÄ
}

_ Nach $₫ 36$ des Bundesmantelvertrags (BMV-Ä) sind Vertragsärzte verpflichtet, den Krankenkassen auf Verlangen alle schriftlichen Informationen $\mathrm{zu}$ übermitteln, die diese für ihre Arbeit brauchen. Das sind etwa Auskünfte, Bescheinigungen, Zeugnisse, Berichte oder Gutachten. In allen Fällen sind hierzu Vordrucke vereinbart. Für derlei Auskünfte gibt es kein besonderes Honorar, nur eine Auslagenerstattung - es sei denn, dass eine andere Vergütungsregelung vereinbart wurde.

Vordrucke der Kassen müssen deshalb einen Hinweis darüber enthalten, ob die Abgabe der Information gesondert vergütet wird oder nicht. Gutachten und Bescheinigungen mit gutachterlichen Fragestellungen, für die keine Vordrucke vereinbart wurden, müssen nach den Leistungspositionen des EBM-Abschnitts II 1.6 vergütet werden. Auch wenn kein vereinbarter Vordruck ver- wendet wird, muss die Krankenkasse angeben, aufgrund welcher Bestimmungen des Sozialgesetzbuchs oder anderer Rechtsvorschriften die Übermittlung der Information zulässig ist und ob bzw. wie sie berechnet werden kann.

Wenn eine Krankenkasse ihrem Versicherten hilft, einen Schadensersatzanspruch zu verfolgen, weil dieser einen Behandlungsfehler vermutet, sind Vertragsärzte berechtigt, die erforderlichen Auskünfte zu erteilen, solange eine aktuelle Schweigepflichtentbindung vorliegt.

\section{MMW-KOMMENTAR}

Ärztliche Berichte nach Nr. 01600 EBM (4,07 Euro) und Arztbriefe nach Nr. 01601 (7,72 Euro) sind auch dann abrechnungsfähig, wenn sie per E-Mail erstellt und verschickt werden. Auch dann kann, wie beim Versand per Post oder Telefax, einmal die Nr. 40120 (0,55 Euro) berechnet werden. Die Berechnung der Nr. 01600 ist allerdings ausge-

\section{Heilmittelverordnung digital!}

\begin{abstract}
- Ab 2017 darf für die Verordnung von Heilmitteln nur noch eine von der KBV zertifizierte Software verwendet werden. Diese muss laut Gesetz dann alle wichtigen Regelungen und Informationen zur Heilmittelverordnung enthalten. Konkret geht es um die Verordnung von Krankengymnastik, Ergotherapie, Fußpflege und Logopädie.
\end{abstract}

\section{MMW-KOMMENTAR}

Schon heute müssen beim Verordnen von Heilmitteln komplexe Vorgaben beachtet werden. Künftig soll zur Entlastung der Praxen die Software deshalb die wichtigen Informationen der Heilmittelrichtlinie enthalten und über besondere Versordnungsbedarfe informieren. Die Software soll Ärzten helfen, die Vorgaben der Heilmittelrichtlinie korrekt umzusetzen und fehlerfrei zu arbeiten. Die Informationen sind dann elektronisch hinterlegt, ein Nachschlagen im Heilmittelkatalog oder in Diagnoselisten entfällt. Die Softwarehäuser haben nun ausreichend Zeit zu programmieren, die KBV-Zertifizierung zu beantragen und die Updates zu liefern. schlossen, wenn im selben Quartal die Versichertenpauschale nach Nr. 03000 abgerechnet wird.

Bei Auftragsleistungen, also ärztlichen Leistungen, die nach Art und Umfang konkret definiert sind, ist die unkommentierte Mitteilung eines Befundes nicht gesondert berechnungsfähig. Dies gilt auch für die Anforderung von Befundmitteilungen und Krankenblättern durch einen ambulant behandelnden Vertragsarzt sowie die Rücksendung solcher Unterlagen.

Werden Briefe, Faxbriefe und E-Mails an Patienten geschickt, können weder Gebührennummern noch Kosten abgerechnet werden. In diesem Fall ist aber eine Privatliquidation nach GOÄ möglich. In Betracht kommen hier die Bescheinigung nach Nr. 70 (Einfachsatz: 2,33 Euro), der Brief nach Nr. 75 (Einfachsatz: 7,58 Euro) oder das Gutachten nach Nr. 80 (Einfachsatz: 17,49 Euro) - jeweils zuzüglich der tatsächlich entstandenen Porto- und Kopierkosten.

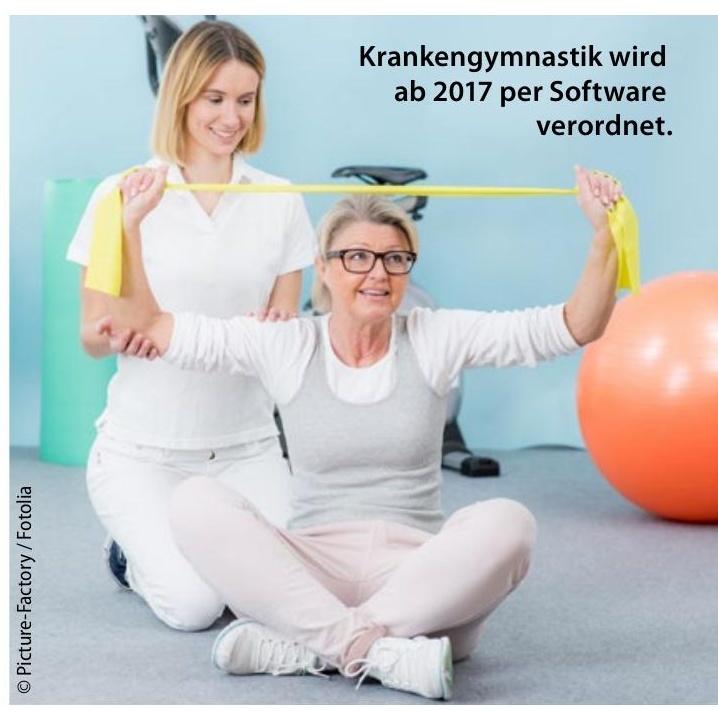

